\title{
A Th2-score in the tumor microenvironment as a predictive biomarker of response to BCG in patients with non-muscle invasive bladder carcinoma. A retrospective study.
}

\section{Gustavo Martín Villoldo}

Urology Department, Instituto Alexander Fleming

María Teresa Pombo

Immunohistochemistry Department, Instituto Alexander Fleming

Mariana Aris

Centro de Investigaciones Oncológicas Fundación Cáncer Joaquín Chemi

Urology Department, Instituto Alexander Fleming

Pablo Mandó

Centro de Investigaciones Oncológicas Fundación Cáncer

\section{Supriya Nagaraju}

Urology Department, MD Anderson Cancer Center

\section{Juan Camean}

Urology Department, Instituto Alexander Fleming

\section{Adrián Burioni}

Urology Department, Instituto Alexander Fleming

\section{Deborah Egea}

Urology Department, Instituto Alexander Fleming

\section{Mora Amat}

Pathology Department, Instituto Alexander Fleming

José León Mellado

Centro de Investigaciones Oncológicas Fundación Cáncer José Mordoh

Centro de Investigaciones Oncológicas Fundación Cáncer Alberto Villaronga

Urology Department, Instituto Alexander Fleming

María Marcela Barrio ( $\sim$ barrio.marcela@gmail.com )

Centro de Investigaciones Oncológicas Fundación Cáncer 


\section{Research Article}

Keywords: non-muscle invasive bladder cancer, BCG, Predictive biomarkers lymphocyte polarization

Posted Date: March 23rd, 2022

DOI: https://doi.org/10.21203/rs.3.rs-1369739/v2

License: (c) (i) This work is licensed under a Creative Commons Attribution 4.0 International License. Read Full License

Version of Record: A version of this preprint was published at Oncology Research on April 10th, 2023. See the published version at https://doi.org/10.32604/or.2023.028163. 


\section{Abstract}

Intravesical BCG is the gold standard therapy for intermediate/high-risk NMIBC. However, the response rate is $~ 60 \%$, and $50 \%$ of non-responders will progress to muscle invasive disease. BCG induces massive local infiltration of inflammatory cells (Th1), and ultimately cytotoxic tumor elimination. We searched for a predictive biomarker of BCG response by analyzing TILs polarization in the TME in pre-treatment biopsies. Materials and Methods: Pre-treatment biopsies from patients with NMIBC who received adequate intravesical instillations of BCG $(n=32)$ were evaluated retrospectively by immunohistochemistry. TME polarization was assessed by quantifying the T-Bet+ (Th1) and GATA-3+ (Th2) lymphocyte ratio (G/T), and the density and degranulation of EPX+ eosinophils. In addition, PD1/PD-L1 staining was quantified. The results correlated with the BCG response. In most nonresponders, Th1/Th2 markers were compared in pre-and post-BCG biopsies.

ORR was $65.6 \%$ in the study population. BCG responders had a higher $\mathrm{G} / \mathrm{T}$ ratio and higher number of degranulated EPX+ cells. Variables combined into a Th2-score showed a significant association with higher scores in responders $(p=0.027)$. A Th2-score cut-off value $>48.1$ allowed discrimination of responders with $91 \%$ sensitivity, but with lower specificity. Relapse-free survival was significantly associated with the Th2-score ( $p=0.007)$. In post-BCG biopsies from recurring patients, TILs increased Th2-polarization, probably reflecting BCG failure to induce a pro-inflammatory status and, thus, a lack of response. PD-L1/PD-1 expression was not associated with the BCG response.

Our results support the hypothesis that a pre-existing Th2-polarized TME predicts a better response to $B C G$, assuming a reversion to Th1 polarization and antitumor activity.

\section{Introduction}

Non-muscle invasive bladder cancer (NMIBC) represents $70 \%$ of all initial bladder cancer diagnoses [1, 2]. Some high-risk patients (high-grade (HG) Pt1 or carcinoma in situ (Cis)) [3] would respond to bladderconservative measures and behave in a non-lethal fashion, while others are aggressive and tend to progress to muscle invasion and even metastatic disease. pT1 tumors are mostly HG and progress in > $50 \%$ of cases; deaths occur in $25 \%$ of patients during the first five years [3]. Cis is mostly concomitant, with increasing recurrence rates from $43-73 \%$ [4]. Even if Cis is primary or concomitant, $50 \%$ progress to muscle invasion and $20 \%$ of patients will ultimately die of metastases if a radical cystectomy is not performed [5]. Intravesical administration of live- attenuated Bacillus Calmette-Guérin (BCG) is the primary adjuvant treatment for high-risk NMIBC patients [6]. Nonetheless, response rate (RR) to BCG is approximately $60 \%$, with a 5-year recurrence rate of 30-40\% [7]. Moreover, for those patients either with pT1, Cis or both tumors, who do not respond to BCG, the risk of progression to invasive disease may reach $50 \%$ [8]. Thus, there is still an important need to find appropriate predictive biomarkers of response to BCG that may help selection of patients with a low chance of response, before or early after treatment starts, to offer them alternative therapeutic options. Although the exact mechanism of BCG antitumor activity is not fully understood, it is recognized that BCG acts as a localized immunomodulatory agent 
towards Th1 immune polarization and its efficacy must be related to the immune response elicited in the bladder [9].

GATA binding protein 3 (GATA-3) is a transcription factor that is critical for T cell development and Th2 differentiation [10]. T-box transcription factor 21 (T-Bet) is mostly expressed in CD4 + Th1 cells, and its expression is induced by a combination of T cell receptor and IFN-y signaling [11]. In addition, T-Bet promotes Th1 polarization by preventing GATA-3 mediated Th2 cell development [12].

In the past, eosinophils were inaccurately considered as destructive end-stage effector cells (helminth infections and allergy/asthma) due to the release of toxic cationic granule proteins and the production of reactive oxygen species. More recently, eosinophils have been shown to play a fundamental role in remodeling/repair activities, and more importantly, to modulate local immunity [13].

One emerging concept relates to the prognostic and/or predictive value of Th1/Th2 polarization of tumorinfiltrating cells (TILs) previous to treatment, which may affect the response to BCG immunotherapy. Interestingly, in patients with Cis treated with BCG induction alone [14], an increased density of intratumor Th2 cells and a higher Th2/Th1 ratio prior to treatment was found in patients who responded to BCG, suggesting that BCG would be effective only when the tumor microenvironment (TME) shifted from Th2 to Th1 immune status. Programmed death ligand 1 (PD-L1) expressed by tumor and immune cells in the TME suppresses antitumor immunity and promotes tumor progression [15]. However, PD-L1 + TILs have been correlated with improved OS in patients with metastatic urothelial cancer [16]. In a recent study, BCG response was not associated with peritumoral PD-L1 expression in pretreatment biopsies [17]. On the contrary, high PD-L1 expression in BCG-induced granulomas is associated with resistance to therapy in urothelial carcinomas [18]. These data suggest that high numbers of PD-L1 + cells in the bladder could suppress the effectiveness of BCG, a hypothesis also supported by the recent demonstration that combined BCG and anti-PD-L1 treatment increased antitumor immunity in an immunocompetent orthotopic rat bladder cancer model [19].

The aim of our study was to identify potential biomarkers to predict the response to BCG in a high-risk population of NMIBC patients, including those with HG pT1 and Cis tumors, focusing on Th1/Th2 lymphocyte polarization in the TME.

\section{Materials And Methods}

\section{Patients and BCG immunotherapy}

This retrospective review of the bladder cancer registry and biopsies from the Urology Department of the Instituto Alexander Fleming (Buenos Aires, Argentina) included patients who were diagnosed between 2007 and 2019. Archive tumor samples were analyzed in this study, and all patients provided written informed consent for the use of biopsies for research purposes at the time of surgery. Patients with NMIBC were stratified according to the EAU risk stratification guidelines [20]. We analyzed the initial bladder tumor biopsy obtained via complete transurethral resection (TURB) and post-treatment biopsies 
when available. Re-TURB was performed when pT1 NMIBC was detected during the first TURB. The patients did not receive any previous treatment before surgery. Adjuvant treatment started 3-4 weeks after TURB and consisted of a standard 6-dose induction course plus a 3-dose maintenance course at 3, $6,12,18,24,30$, and 36 months of BCG (120 mg of Danish strain $1331 \mathrm{SSI})$ [21]. The patients did not receive any other intravesical therapy during or after TURB. Follow-up cystoscopy, cytology, and TC scans were performed according to the guidelines [20]. Patients were classified as nonresponders (NR) if they had a positive biopsy result for bladder carcinoma after BCG induction plus one maintenance course (adequate BCG). BCG responders (R) were defined as those without any recurrence or evidence of disease based on follow-up cystoscopy, urinary cytology, or re-biopsy. Patients with a history of immunosuppressive drug treatment or other possible confounding factors were also excluded.

\section{Immunohistochemical staining}

The biopsies were fixed in formalin and paraffin embedded. Paraffin blocks containing sufficient material were selected for immunohistochemical (IHC) staining with the following anti-human monoclonal Abs: eosinophil peroxidase EPX-mAb (clone MM 25-82.2, kindly provided by Mayo Clinic), GATA-3 (clone L50823, Cell Marque), T-Bet (clone EPR9302 RabMab, Abcam), and desmin to confirm muscle indemnity (clone D33, Dako). The sections were stained with anti-human PD-L1 (clone SP263, Ventana) and antihuman PD-1 (clone NAT105, Abcam). All markers, except PD-L1, were determined using standardized automated protocols for LEICA BOND MAX II. PD-L1 expression was determined using the Benchmark ULTRA (Roche, Basel, Switzerland). Sections were examined by optical microscopy (Olympus BX40 microscope, DP2-BSW software), and digitalized images were analyzed using ImageJ software (NIH).

\section{Quantitation and scoring}

For each immune population, IHC staining was quantified as the average of positive cells in eight highmagnification fields (400x) covering a $1.2 \mathrm{~mm} 2$ area. EPX + eosinophils were quantified at the maximum focus of eosinophilic infiltration (Eo count). Also, EPX-mAb staining was used to identify the maximum level of eosinophilic degranulation (Eodgn). A numerical value was given for each level of eosinophil degranulation based on the normal distribution of the data as follows: $0=$ no degranulation, $1=1 \mathrm{a}$, small sharp granules or $1 \mathrm{~b}$, granules with fuzzy edges, and $2=$ multiple granules of imprecise edges far from the cell body. GATA-3 + and T-Bet + lymphocytes were quantified at the maximum focus of mononuclear cell infiltration. The ratio of Th2 polarized (GATA-3+)/Th1 polarized (TBet+) lymphocytes (G/T) was calculated. A Th2-score was defined as:

Th2-Score $=\mathrm{G} / \mathrm{T}+\left(\right.$ Eo counts ${ }^{\star}$ Eodgn $)$

PD-L1 expression was quantitated by the combined positive score (CPS), which is the number of PD-L1 + cells (tumor cells, lymphocytes, macrophages) divided by the total number of tumor cells, $x 100$ ).

Percentages of $>1 \%$ were considered positive for PD-L1. To control for inter-observer variability, all IHC 
counts were performed in duplicate by two double-blinded investigators. Investigator 1 counts were used because difference between counts was $<10 \%$.

\section{Statistical analysis}

Independent categorical variables were described as percentages and compared using Fisher's exact test or the chi-square test. Comparisons between R and NR patients were performed using the nonparametric Mann-Whitney U test. Spearman's correlation coefficient was used to identify associations between continuous variables. GraphPad Prism v10 (GraphPad Software Inc., La Jolla, CA, USA) was used for all statistical analyses. The predictive power of biomarker levels on the BCG response was evaluated by plotting receiver operating characteristic (ROC) curves and calculating the area under the curve (AUC). Recurrence-free survival (RFS) was compared using the Kaplan-Meier method, stratifying patients by the Th2-score and other variables with the log-rank test using R. $p$ values were two-sided, and $p<0.05$ was considered statistically significant.

\section{Results}

\section{Response to BCG treatment}

In total 32 patients were included in this retrospective study (Table 1). The mean age at diagnosis was 64.1 years overall. Histopathological evaluation confirmed primary high-risk NMIBC according to the EAU guidelines. Six patients showed LG, while 26 (81. 2\%) had a HG cancer, according to the WHO 2004/2016 classification. Most patients had pT1 (24/32) and pTa tumors (6/32). Two patients had Cis only, and two patients had pT1HG + Cis and pTa LG + Cis. One patient had concurrent prostate cancer that was treated with radical prostatectomy at the time of NMIBC diagnosis.

The median follow-up period for the patients without recurrence was 37 months. The pathological finding at post-BCG control was at pT0 in 24 patients. overall response rate (ORR) to BCG treatment was 65.6\%, as $21 / 32$ patients remained recurrence free for at least 20 months. BCG NR had a median time to recurrence of 15 months, however 7/11 (63.3\%) recurred before 12 months. RR was not significantly associated with sex, age, tumor stage, or histological grade in the study population.Of the 11 patients who experienced BCG failure, 3 progressed to muscle-invasive disease.

\section{Tumor infiltrating lymphocyte's profile}

The expression of T-Bet and GATA-3 was detected in the TILs of all pre-BCG treatment biopsies (Figure 1a). Additionally, eosinophils infiltrating the tumor area were detected in 31/32 biopsies (median Eo count 5.8; range 0-96.5). The extent of Eo degranulation (Eodgn), as a readout of its activation in the TME, could be evaluated as EPX+ granules were clearly evident. A significantly higher infiltration of Th2 cells than Th1 cells was identified, with a median (range) number of total GATA- 3+ and T-Bet+ cells of 21.5 (0.5-194) and $2.9(0.125-134)$, respectively $(p<0.0005)$ (Supplementary Table 1 and Figure 1b). 
No significant correlation was found between the total number of GATA-3+ and TBet+ cells, GATA-3+ and Eo counts, or Eodgn or Tbet+ and EPX+ cells or Eodgn. Only Eo counts correlated with Eodgn, suggesting that when eosinophils were abundant in the TME, most were activated (Supplementary Table 2).

TIL expression of GATA-3, T-Bet, and EPX (Eo counts or Eodgn) was compared in biopsies from BCG-R and-NR. The BCG response was associated with a higher number of GATA-3+ and EPX+ cells, a higher GATA-3/T-bet ratio (G/T), and a lower number of T-Bet+ cells, although these differences were not statistically significant (Supplementary File 1, Figure 1).

\section{Th2 Score predicts BCG response}

When we combined the G/T ratio (TIL polarization) and the product of Eo counts and Eodgn, the BCG response was significantly associated with a higher Th2-score $(p=0.0277)$ (Figure 2a).

The AUC of the pre-BCG Th2-score was 0.74 ( $95 \% \mathrm{Cl} 0.56-0.91 ; p=0.028)$. Since the aim of the predictive score would be to identify true R to offer BCG treatment with high chances of success, a cutoff value for the Th2-score was set at $>48.1$, allowing discrimination of R with $91 \%$ sensitivity; however specificity was $57.1 \%$ (PPV=0.526, NPV= 0.923) (Figure 2b).

For survival analyses, we divided the patients into two groups (low vs. high counts) based on the median values of the different variables analyzed ( 65.5 for age; 21.5 for GATA-3+; 2.9 for T-Bet+; 3.6 for G/T and 5.9; EPX+) (Supplementary Table 1). No significant association was found for the other individual variables (GATA-3+, T-Bet+ cells, or G/T). Patients with high Eo counts tended to have a longer RFS than those with low Eo counts $(p=0.052)$ (Supplementary File 1, Figure 2$)$. Then, the patients were split into "high-Th2" and "low-Th2" score subgroups according to the cutoff value (48.1). Patients with a high Th2score on pre-BCG biopsy had a significantly prolonged RFS compared with those with a low Th2-score $(p=0.007)$ (Figure $2 c)$.

Pre-BCG biopsies were compared to post-BCG biopsies in most cases of NR $(n=9)$. The median time to recurrence was 8 months. Of note, in $8 / 9$ tumors $(88.9 \%)$, GATA-3+ TILs increased after recurrence $(p=$ 0.055) (Figure $2 \mathrm{~d}$ ). Accordingly, the $\mathrm{G} / \mathrm{T}$ and Th2-score were also higher in $7 / 9$ post-BCG biopsies (77.8\%) as compared to pre-BCG tumors; however, these differences were not statistically significant (Figure 2 e,f).

\section{PD-1 and PD-L1 expression in NMIBC biopsies and response to BCG}

Due to limited tumor availability, PD-L1/PD-1 staining was evaluated in 20 and 19 biopsies, respectively. In pre-BCG biopsies, PD-L1+ tumor cells were observed only in $8 / 20$ tumors (40\%), but could be detected in TILs in 15/20 biopsies (75\%) a higher proportion of tumors from R expressed PD-L1 in $>5 \%$ of TILs than in NR $(p=0.051)$ (Figure $3 \mathbf{a}, \mathbf{b})$. PD-L1+ tumor cells were correlated with PD-L1+ TILs in all pre-BCG biopsies $(r=0.68, p=0.0014)$, but these values were not associated with BCG response (Figure $3 c)$. PD-1+ TILs $>50 \%$ were found in $4 / 12$ biopsies from BCG R, but only in 1/7 of NR, although this difference was not statistically significant (Figure 3d). Representative images of PD-L1 and PD-1 expression in patients with R and NR are shown in Figure 3e. PD-1+ or PD-L1+ TILs were not correlated with GATA-3 or T-Bet 
expression or with the Th2-score ( $p>0.05)$, independent of the BCG response. In post-BCG samples, tumor cells and TILs showed no PD-L1 expression (0-3\%), and PD-1+ TILs were $<1 \%$ (Supplementary Table 1).

\section{Discussion}

There is a concrete need to identify patients with NMIBC who will not benefit from BCG immunotherapy to offer them alternative treatments or clinical trials. In vitro and in vivo evidence supports that BCG acts as a potent Th1 inducer in the TME and is presumably responsible for long-lasting disease control $[9,22]$. However, which is the nature of the targeted tumor antigens or whether the anti-BCG response is involved in tumor elimination is still unknown. Additionally, experimental evidence has shown a direct effect of BCG in killing bladder cancer cells [23].

In the search for a possible biomarker of response to BCG, in pre-BCG biopsies of patients with high-risk NMIBC who received BCG, we evaluated lymphocyte polarization in the TME along with the quantification of eosinophils (associated with a Th2 immune microenvironment) by IHC. Our goal was to define a Th2score associated with BCG response and set a cutoff to predict true BCG R with $>90 \%$ sensitivity. Patients with higher Th2-score showed significantly higher RFS after BCG treatment.

Nunez-Nateras et al [14] reported that a Th2-score obtained after quantitation of the Th2/Th1 lymphocyte ratio and eosinophilic infiltration in the TME of pre-BCG biopsies was associated with BCG response in 38 Cis NMIBC patients. The authors hypothesized that a pre-existing Th2 TME would be susceptible to Th1 polarization and therefore correlate with the response to BCG treatment. However, in that study, patients received only one course of induction $\times 6$, which is inadequate today. Another study confirmed the findings of Nunez-Nateras's findings [24]. However, the small number of patients included (19 BCG R and $4 \mathrm{NR}$ ) may limit the conclusions, and the fact that only $25 \%$ of the patients had Cis makes difficult comparisons. Another report on HG PT1 patients showed that BCG R had a significantly lower level of peritumoral TBet + cells and a higher G/T ratio [17]. Taken together, these results strongly suggest that, after surgery, BCG can eradicate NMIBC tumors only when the TME converts from Th2 to Th1. Interestingly, we observed an increase in Th2 TILs in post-BCG biopsies from relapsed patients, probably reflecting the failure of BCG to induce a pro-inflammatory status. Presumably, residual tumor cells that established strong immunosuppression sustained Th2 polarization.

Emerging data highlight that eosinophils infiltrate multiple tumors and display pleotropic and even opposing roles (pro- vs. anti-tumorigenic activities) [25]. Furthermore, crosstalk between activated eosinophils and T cells increases CD8 + T cell migration to TME [26]. In addition, in patients treated with immune checkpoint inhibitors, increased eosinophilia is associated with responsiveness to therapy [26]. Thus, a higher pre-BCG Eo count in R could represent a Th2 polarized TME that can be shifted to Th1 after BCG administration, and eosinophils may contribute to Th1 cell recruitment. However, it has been reported that blood eosinophils in patients with NMIBC could predict disease recurrence during BCG immunotherapy [27]. More data are needed to clarify this idea. 
Immune checkpoint upregulation is associated with a lack of antitumor cytotoxicity [28]. The upregulation of PD-L1 allows tumor cells to escape immune surveillance; thus, PD-L1 expression in the pretreatment TME could be a mechanism of BCG resistance. In our study, PD-L1 + tumor cells in pre-BCG biopsies were not associated with BCG response, in accordance with a previous report [17]. However, a higher proportion of tumors from R had $>5 \%$ PD-L1 + TILs than those from NR. Our findings are in disagreement with another report that found elevated PD-L1 expression in CD8 + TILs in NR [29]. However, we used a different anti-PD-L1 antibody (Ventana clone SP263 instead of Spring Biosciences clone SP142 or DAKO clone 22C3), and fewer patients were analyzed. These results should be confirmed in larger cohorts of patients. We observed $>50 \%$ PD-1 + TILs in a higher proportion of R than of NR, although this difference was not statistically significant, probably due to the small number of cases analyzed. No exhausted PD-1 + TILs were detected in post-BCG biopsies of relapsed patients. One possible explanation could be that prior to BCG, an antitumor immune response was generated in some patients, accounting for the detection of exhausted T cells. However, a Th2 TME induced by the tumor provides a cytokine balance that prevents the cytotoxic activity of TILs. In addition, PD-L1 + cells in the TME could contribute to the further abrogation of the antitumor response. Treatment with BCG shifts this balance towards Th1 polarization in $\mathrm{R}$, increasing the affluence of innate and adaptive immune cells. Th1 cytokine production, mainly IFN- - , may fuel the cytotoxic elimination of residual tumor cells. Instead, in pre-BCG tumors with Th1-profiled TILs, other immunosuppressive mechanisms, such as secretion of TGF- $\beta$, PGE2, IL-10, and IL-6, and accumulation of myeloid-derived suppressor cells, tumor-associated macrophages, and regulatory $T$ cells, could contribute to creating a highly tolerogenic TME [30]. Due to the small number of tumors available, we were not able to evaluate these immune populations in pre-BCG biopsies.

This retrospective study was limited to the analysis of fixed tumor tissues using $\mathrm{IHC}$. We are starting a prospective study including samples of peripheral blood cells and urine obtained before and throughout BCG immunotherapy to initiate future research about the immune mechanisms underlying BCG treatment for NMIBC. Our results suggest that immune cells present in the pre-BCG TME can influence the BCG response and that our Th2-score could help identify patients who might benefit from BCG therapy.

\section{Abbreviations}

AUC, area under the curve

BCG, Bacillus Calmette-Guérin

Cis, carcinoma in situ

Eo, eosinophil

Eodgn, eosinophilic degranulation

G/T, GATA-3+/T-Bet + ratio

HG

high grade

IFN- $\mathrm{Y}$ interferon gamma

$\mathrm{IHC}$ 
immunohistochemical staining

LG

low grade

NMIBC

non-muscle invasive bladder cancer

NR

BCG Non-Responder

ORR

Overall response rate

$\mathrm{R}$

BCG responder

RFS

recurrence-free survival

ROC

receiver operating characteristic

RR

response rate

TILs

tumor infiltrating lymphocytes

TME

tumor microenvironment

TURB

transurethral resection of bladder tumor

\section{Declarations}

\section{Ethics approval and consent to participate}

This retrospective review of the bladder cancer registry and biopsies from the Urology Department of the Instituto Alexander Fleming (Buenos Aires, Argentina) included patients who were diagnosed between 2007 and 2019. In this study, archived tumor samples were analyzed, and all patients signed an informed consent form for the use of biopsies for research purposes at the time of surgery. All the procedures were performed as part of the routine care, in accordance with the relevant guidelines and regulations of the Instituto Alexander Fleming. This retrospective study was approved by the Ethics Committee of Instituto Alexander Fleming (CEIAF) under resolution \#532. CEIAF approved a waiver of further informed consent signing, in view of the retrospective nature of the study and the lack of identifiable patient s information included.

\section{Consent to publication}

Nor applicable 
Availability of data and materials

All data generated or analysed during this study are included in this published article and its supplementary information files.

\section{Competing interests}

The authors have no competing interests to declare that are relevant to the content of this article.

\section{Funding}

This work was supported by grants from CONICET, Agencia Nacional de Promoción Científica y Técnica (PICT 2018 00990, FONCYT) de Argentina, Fundación Sales, Fundación Pedro Mosoteguy and Fundación Cáncer FUCA

\section{Author Contributions}

Gustavo Villoldo and Maria Marcela Barrio contributed to the study conception and design. Material preparation and data collection was performed by Joaquín Chemi, Juan Camean, Adrián Burioni Deborah Egea, Mora Amat and Alberto Villaronga. Immunohistochemistry staining and quantitation was performed by María Teresa Pombo and Mariana Aris. Data analysis and statistics was performed by Pablo Mandó, Supriya Nagaraju and José Mordoh. José León Mellado contributed to preparation of figures and manuscript edition. The first draft of the manuscript was written by María Marcela Barrio and all authors commented on previous versions of the manuscript. All authors read and approved the final manuscript.

\section{Acknowledgements}

We dedicate this work to our patients.

\section{Authors' information}

Gustavo Villoldo, Alberto Villaronga, Joaquín Chemi and Deborah Egea are surgeons at the Urology Department of the Instituto Alexander Fleming.

\section{References}

1. Fleshner NE, Herr HW, Stewart AK et al (1996) The National Cancer Database report on bladder carcinoma. The American College of Surgeons Commission on Cancer and the American Cancer Society. Cancer. 78:1505-1513. https://doi.org/10.1002/(SICI)10970142(19961001)78:7<1505::AID-CNCR19>3.0.C0;2-3

2. Sylvester RJ, van der MA, Lamm DL (2002) Intravesical bacillus Calmette Guerin reduces the risk of progression in patients with superficial bladder cancer: a metaanalysis of the published results of 
randomized clinical trials. J Urol 168: 1964-1970.

https://doi.org/10.1097/01.ju.0000034450.80198.1c

3. Herr HW (1997) Tumour progression and survival in patients with T1G3 bladder tumours: 15-year outcome. Br J Urol. 80: 762-765. https://doi.org/10.1046/j.1464-410X.1997.00431.x

4. Kiemeney LA, Witjes JA, Heijbroek RP et al (1994) Dysplasia in normal-looking urothelium increases the risk of tumour progression in primary superficial bladder cancer. Eur J Cancer. 30A: 1621-1625. https://doi.org/ 10.1016/0959-8049(94)e0133-o

5. Jakse G, Hall R, Bono A et al (2001) Intravesical BCG in patients with carcinoma in situ of the urinary bladder: long-term results of EORTC GU Group phase II protocol 30861. Eur Urol. 40:144-150. https://doi.org/10.1159/000049765

6. Herr HW, Morales A (2008) History of bacillus Calmette-Guerin and bladder cancer: an immunotherapy success story. J Urol 179: 53-56. https://doi.org/10.1016/j.juro.2007.08.122

7. Griffiths TR, Charlton M, Neal DE et al (2002) Treatment of carcinoma in situ with intravesical bacillus Calmette-Guerin without maintenance. J Urol 167:2408-2412. https://doi.org/10.1016/S0022-5347(05)64994-4

8. Witjes JA (2006) Management of BCG failures in superficial bladder cancer: a review. Eur Urol 49: 790-797. https://doi.org/10.1016/j.eururo.2006.01.017

9. Kamat AM, Briggman J, Urbauer DL et al (2016) Cytokine panel for response to intravesical therapy (CyPRIT): nomogram of changes in urinary cytokine levels predicts patient response to bacillus Calmette-Guérin. Eur Urol 69: 197-200. http://doi.org/10.1016/j.eururo.2015.06.023

10. Zeng WP (2013) 'All things considered': transcriptional regulation of T helper type 2 cell differentiation from precursor to effector activation. Immunology. 2013; 140: 31-38. https://doi.org/10.1111/imm.12121

11. Lazarevic V, Glimcher LH, Lord GM. T-bet: a bridge between innate and adaptive immunity. Nat Rev Immunol 13: 777-789. https://doi.org/10.1038/nri3536

12. Renkema KR, Lee JY, Lee YJ et al (2016) IL-4 sensitivity shapes the peripheral CD8 + T cell pool and response to infection. J Exp Med 213:1319-1329. https://dx.doi.org/10.1084\%2Fjem.20151359

13. Grisaru-Tal S, Itan M, Klion AD et al (2020) A new dawn for eosinophils in the tumour microenvironment. Nat Rev Cancer 20: 594-607. https://doi.org/10.1038/s41568-020-0283-9

14. Nunez-Nateras R, Castle EP, Protheroe CA et al (2014) Predicting response to bacillus Calmette-Guérin (BCG) in patients with carcinoma in situ of the bladder. Urol Oncol 32: 45.e23-45.e30.. https://doi.org/10.1016/j.urolonc.2013.06.008.

15. Ostrand-Rosenberg S, Horn LA, Haile ST. The programmed death-1 immunesuppressive pathway: barrier to antitumor immunity. J Immunol. 2014; 193: 3835-3841. https://doi.org/10.4049/jimmunol.1401572

16. Bellmunt J, Mullane SA, Werner L et al (2015) Association of PD-L1 expression on tumor-infiltrating mononuclear cells and overall survival in patients with urothelial carcinoma. Ann Oncol 26: 812817. https://doi.org/10.1093/annonc/mdv009 
17. Martínez R, Tapia G, De Muga S et al (2019) Combined assessment of peritumoral Th1/Th2 polarization and peripheral immunity as a new biomarker in the prediction of BCG response in patients with high-risk NMIBC. Oncoimmunology 8: 1602460.

https://dx.doi.org/10.1080\%2F2162402X.2019.1602460

18. Inman BA, Sebo TJ, Frigola X et al (2007) PD-L1 (B7-H1) expression by urothelial carcinoma of the bladder and BCG-induced granulomata: associations with localized stage progression. Cancer 109:1499-1505. https://doi.org/10.1002/cncr.22588

19. Wang Y, Liu J, Yang $X$ et al (2018) Bacillus Calmette-Guérin and anti-PD-L1 combination therapy boosts immune response against bladder cancer. OncoTargets Ther 11: 2891-2899. https://doi.org/10.2147/ott.s165840

20. Sylvester RJ, Rodríguez O, Hernández V et al (2021) European Association of Urology (EAU) Prognostic Factor Risk Groups for Non-muscle-invasive Bladder Cancer (NMIBC) Incorporating the WHO 2004/2016 and WHO 1973 Classification Systems for Grade: An Update from the EAU NMIBC Guidelines Panel. Eur Urol 79: 480-488. https://doi.org/10.1016/j.eururo.2020.12.033

21. Lamm DL, Blumenstein BA, Crissman JD et al (2000) Maintenance bacillus Calmette-Guerin immunotherapy for recurrent TA, T1 and carcinoma in situ transitional cell carcinoma of the bladder: a randomized Southwest Oncology Group Study. J Urol 163: 1124-1129. https://doi.org/10.1016/S0022-5347(05)67707-5

22. Paparo SR, Fallahi P (2017) Bladder cancer and Th1 chemokines. Clin Ter 168: e59-63. https://doi.org/10.7417/ct.2017.1984

23. Yu DS, Wu CL, Ping SY et al (2015) Bacille Calmette-Guerin can induce cellular apoptosis of urothelial cancer directly through toll-like receptor 7 activation. Kaohsiung J Med Sci 31: 391-397. https://doi.org/10.1016/j.kjms.2015.05.005

24. Pichler R, Fritz J, Zavadil C et al (2016) Tumor-infiltrating immune cell subpopulations influence the oncologic outcome after intravesical Bacillus Calmette-Guérin therapy in bladder cancer. Oncotarget 7: 39916-39930. https://doi.org/10.18632/oncotarget.9537

25. Varricchia G, Galdiero MR, Loffredo $S$ et al (2018) Eosinophils: The unsung heroes in cancer? Oncoimmunology 7: e1393134. https://doi.org/10.1080/2162402X.2017.1393134

26. Grisaru-Tal S, Dulberg S, Beck L et al (2021) Metastasis-entrained eosinophils enhance lymphocytemediated anti-tumor immunity. Cancer Res 81: 5555-5571. https:// doi.org/10.1158/00085472.CAN-21-0839.

27. Temiz MZ, Colakerol A, Ulus I et al (2021) Prediction of non-muscle-invasive bladder cancer recurrence during intravesical BCG immunotherapy by use of peripheral blood eosinophil count and percentage: a preliminary report. Cancer Immunol Immunother 70: 245-252. https://doi.org/10.1007/s00262-020-02673-x.

28. Boussiotis VA (2016) Molecular and biochemical aspects of the PD-1 checkpoint pathway. N Engl J Med 375: 1767-1778. https://doi.org/10.1056/NEJMra1514296. 
29. Kates M, Matoso A, Choi W et al (2020) Adaptive Immune Resistance to Intravesical BCG in NonMuscle Invasive Bladder Cancer: Implications for Prospective BCG unresponsive Trials. Clin Cancer Res 26: 882-891. https://doi.org/10.1158/1078-0432.CCR-19-1920.

30. Crispen PL. and Kusmartsev S (2020). Mechanisms of immune evasion in bladder cancer. Cancer Immunol Immunother 69:3-14. https://doi.org/10.1007/s00262-019-02443-4.

\section{Tables}

Table 1 - Clinicopathological parameters associated with BCG immunotherapy response

\begin{tabular}{|c|c|c|c|}
\hline & $\begin{array}{l}\text { BCG responders } \\
n=21\end{array}$ & $\begin{array}{l}\text { BCG non-responders } \\
n=11\end{array}$ & $\mathrm{p}$ value \\
\hline Gender: n (\%) & & & $0.70 *$ \\
\hline Male & $14(71.4)$ & $6(28.6)$ & \\
\hline Female & $7(54.5)$ & $5(45.5)$ & \\
\hline Age (years), median (range) & $66(31-82)$ & $61(52-71)$ & $0.68^{*}$ \\
\hline$\leq 65$ & $8(61.5)$ & $5(38.5)$ & \\
\hline$>65$ & $13(68.4)$ & $6(31.6)$ & \\
\hline Pathological stage: n (\%) & & & $0.14^{\star *}$ \\
\hline $\mathrm{He}$ & $2(33.3)$ & $4(44.7)$ & \\
\hline T1 & $18(75)$ & $6(25)$ & \\
\hline $\mathrm{Cis}^{* * *}$ & $3(75)$ & $1(25)$ & \\
\hline Histological grade: n (\%) & & & $0.15^{\star}$ \\
\hline Low & $2(33.3)$ & $4(66.7)$ & \\
\hline High & 19 (73.1) & $7(26.9)$ & \\
\hline
\end{tabular}

* Fisher's exact test; ** Chi-Square test. ${ }^{* \star *}$ Including both isolated and concomitant Cis in 2 and 2 patients, respectively.

\section{Figures}


A
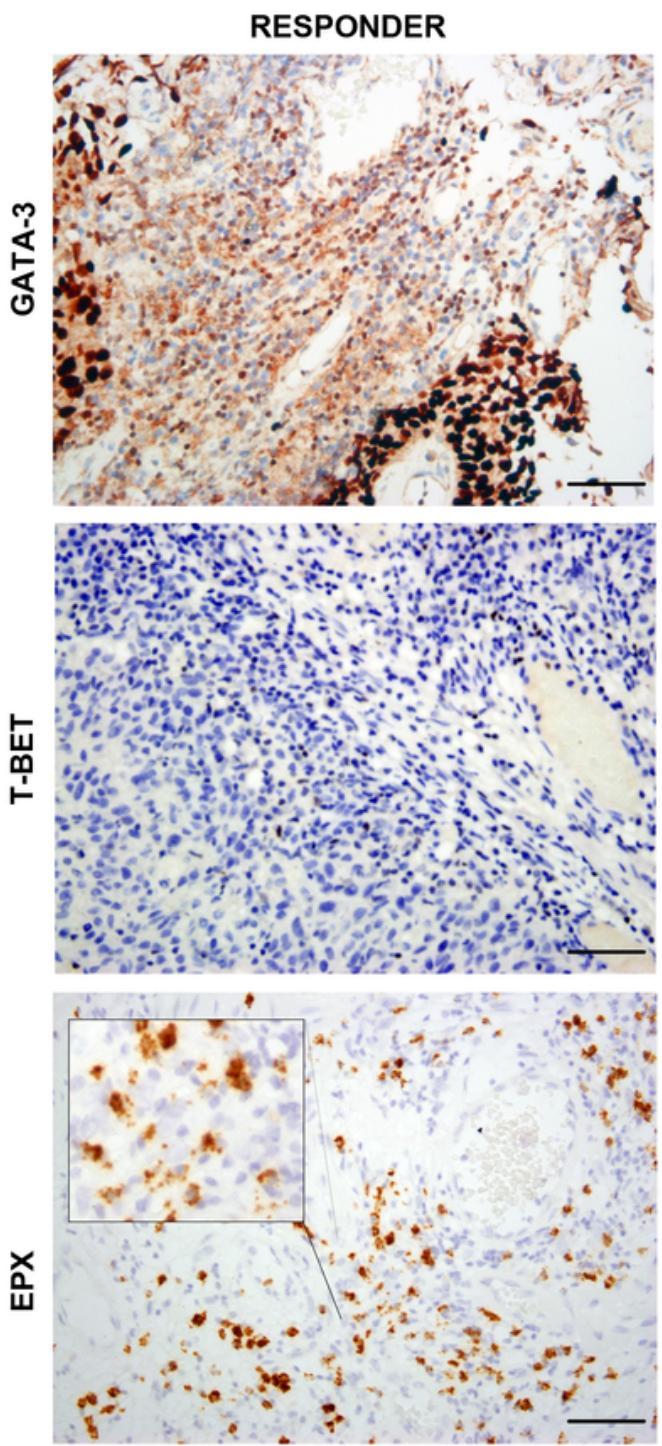

NON-RESPONDER
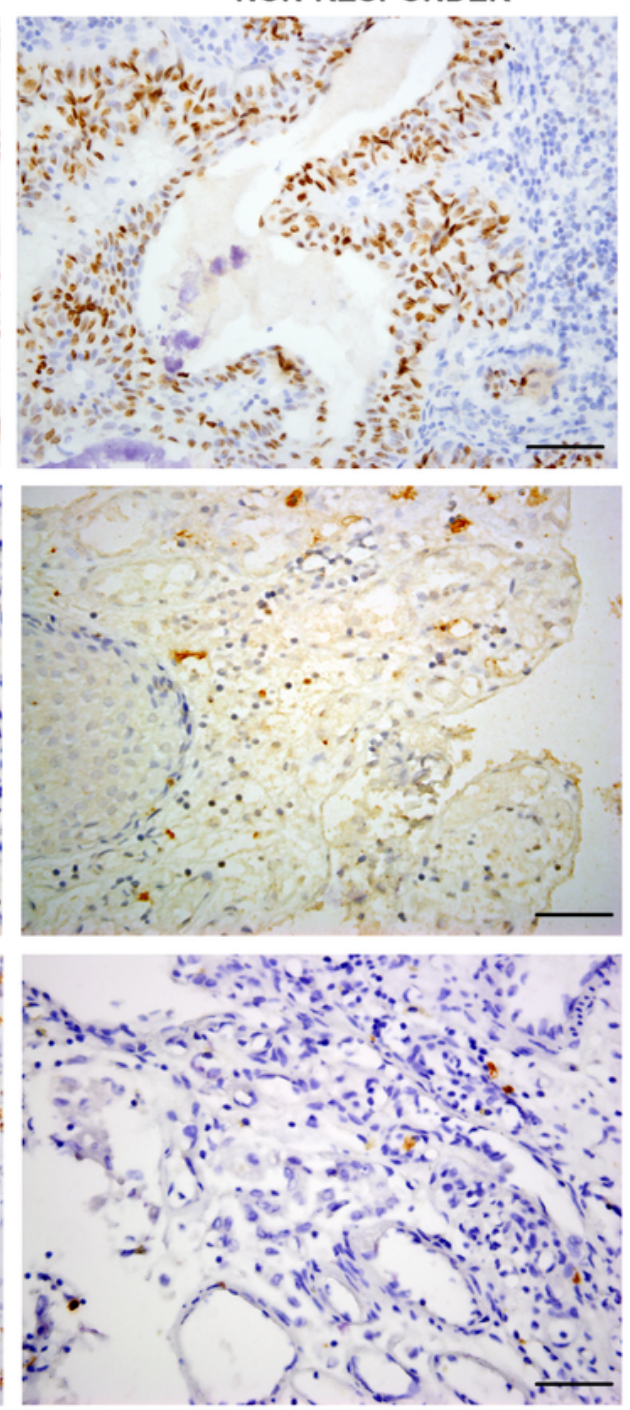

B

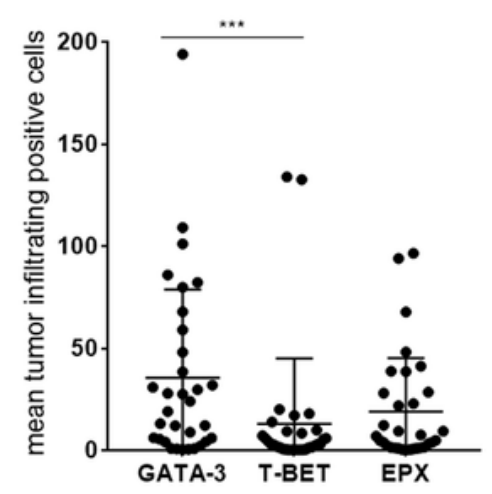

Figure 1

Expression of GATA-3, T-bet and EPX in all NMIBC pre BCG biopsies. a) Representative pictures of immunohistochemistry staining for GATA-3, T-bet and EPX in pre-BCG NMIBC biopsies of R and NR patients. Original magnification 400x. Scale bar $=50 \mu \mathrm{m}$. In case of EPX staining, an inset shows Eodgn in detail. b) Quantitation of GATA-3+, T-bet+ TILs and EPX+ eosinophils EPX in all pre-BCG NMIBC biopsies analyzed. ***highly significant, $p=0.0005$, Mann Whitney test. 
A

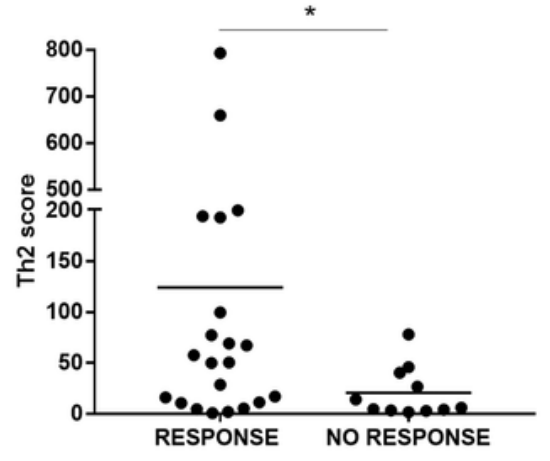

B

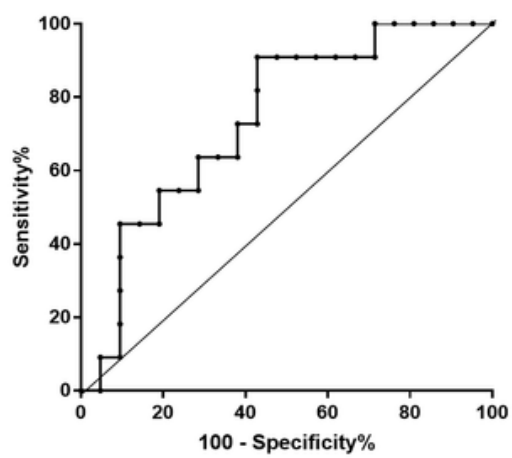

C
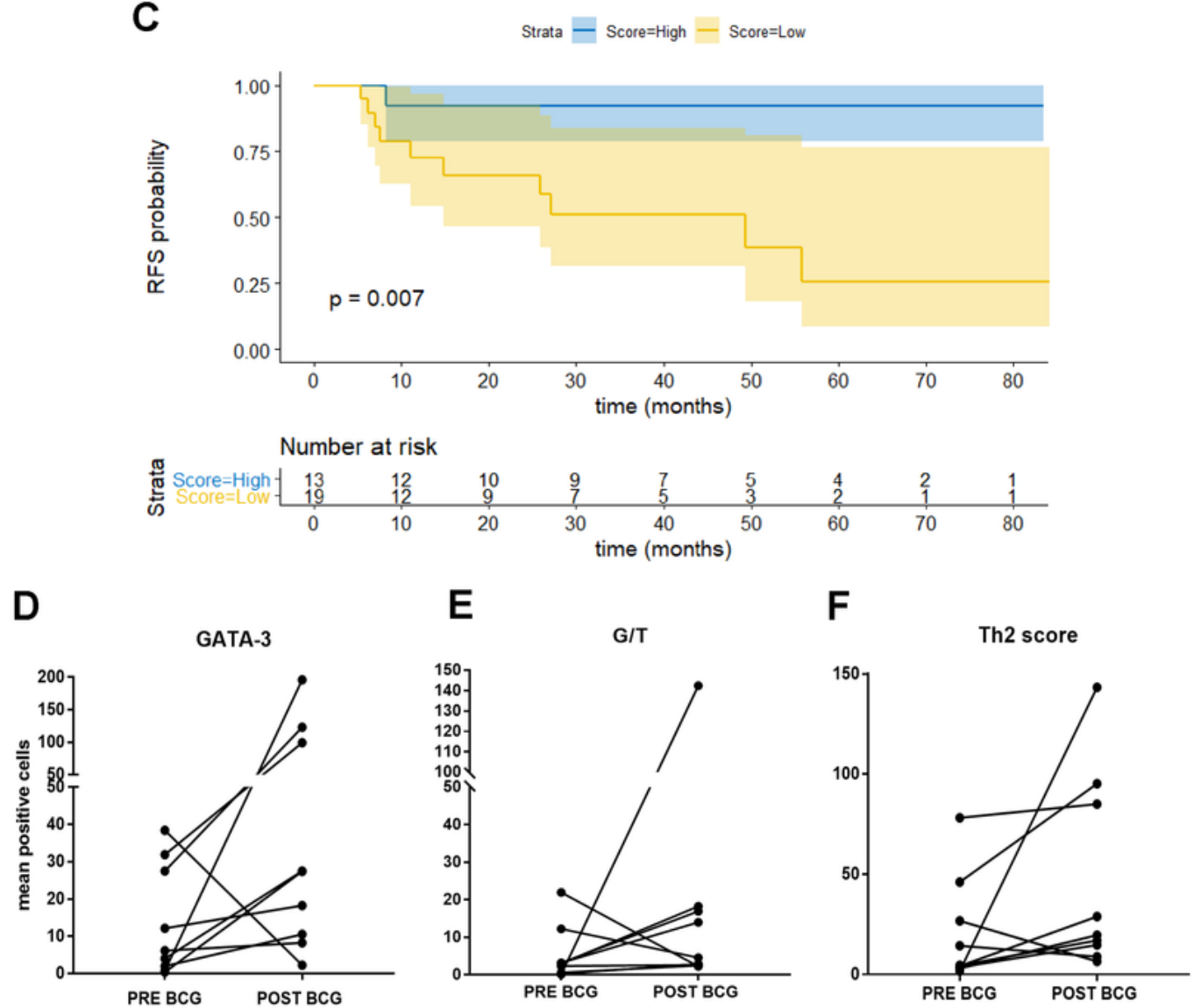

\section{Figure 2}

A Th2-score in the TME can predict response to BCG in NMIBC patients. a) Right: a) Th2-score was higher in BCG R than in NR ( ${ }^{*} p=0.027$, Mann- Whitney test); b) ROC curve for the Th2-score allows discrimination of BCG responding- patients ( $\geq 48.1$ ) with $91 \%$ sensitivity and $57 \%$ specificity. c) Kaplan Meyer curve of recurrence-free survival (RFS) in months. Patients were stratified by Th2-score as low or high, based on the ROC curve cut-off ( $<48.1=$ low count; $\geq 48.1=$ high count) ( $p=0.007$, log rank test (R); $d$, 
e and f) Comparison of GATA-3 mean counts, G/T and Th2-score in pre and post-BCG biopsies (relapses), respectively.
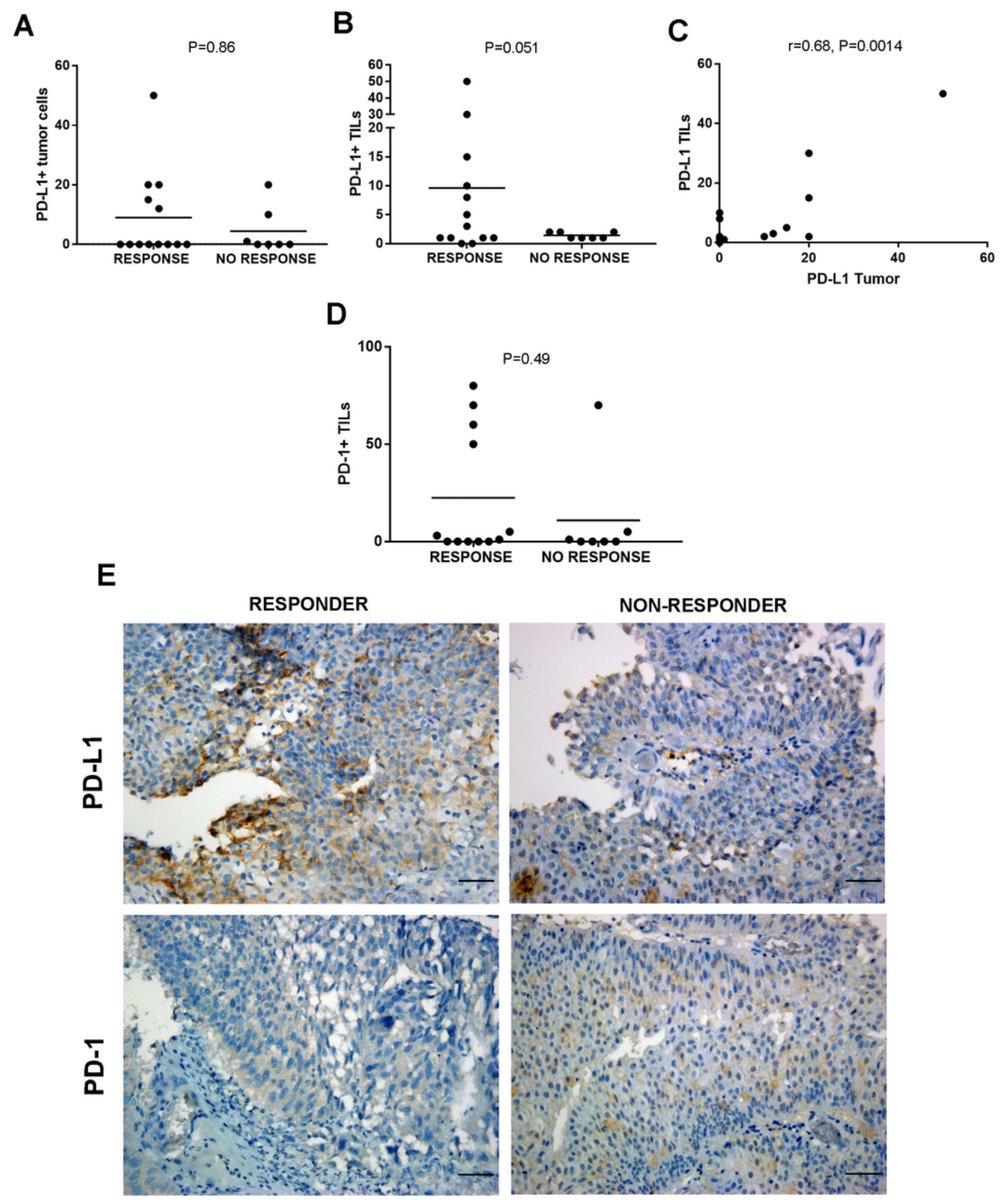

Figure 3

PD-L1/PD-1 expression in pre-BCG TILs of NMIBC biopsies. Quantitation of PD-L1 in tumor cells (a) and TILs (b) performed in BCG R or NR. A positive correlation was found between PD-L1+ tumor cells and PD- 
L1+ TILs (c, r= 0.68, p=0.0014, Spearman correlation test). d) Invasive urothelial carcinoma showing PDL1+ expression at the plasma membranes (left) and stromal TILs expressing PD-L1 in proximity to tumor cells (right). e) Representative pictures of immunohistochemical staining of PD-L1 and PD-1 of pre- BCG tumor biopsies of R and NR patients. Original magnification 400x. Calibration bar 50 $\mu \mathrm{m}$.

\section{Supplementary Files}

This is a list of supplementary files associated with this preprint. Click to download.

- SuplementaryFile1.pdf

- SupplementaryTable1rawdataVilloldoetal2022BMCUrology.xIsx

- SupplementaryTable2.pdf 